\title{
La problemática de los incendios forestales y bases para su teledetección en el Perú
}

\section{The problem of forest fires and the basis for its teledetection in Perú}

\author{
Pamela Lucero Sabuco Cárdenas \\ Universidad Continental
}

\section{INTRODUCCIÓN}

Los incendios forestales se han convertido en un problema ambiental de gran relevancia y gravedad en las últimas décadas, dicho problema se presenta a nivel mundial sobre todo en los países con grandes extensiones de áreas forestales. En América del Sur entre 1998 y 1999, se perdieron un total de 9,4 millones de hectáreas de bosques tropicales a consecuencia de incendios forestales, registrándose alrededor de 219000 incendios y quemas en Sudamérica (1). A nivel nacional, no estamos fuera de este problema; el estado peruano es uno de los países con gran incidencia de incendios forestales, debido a que presenta grandes áreas forestales, pero que en los últimos años hubo un incremento en la perdida de estas áreas, ello relacionado con el crecimiento de actividades socioeconómicas. Solo en el año 2011 hubo un reporte de 26 casos de incendios forestales, esta cifra parecería insignificante, pero cabe aclarar que este número de incendios forestales solo es el reporte de incendios forestales atendidos o de gran magnitud, lo que no representa menos del $25 \%$ de todos los incendios ocurridos, es por ello que el problema de los incendios forestales ha llevado a aumentar el interés y preocupación nacional e internacional asociado con el cambio del clima y con la pérdida de biodiversidad que son temas de interés en diversas convenciones y conferencias internacionales.

Diversas investigaciones afirman que el $99 \%$ de las causas de los incendios es de origen humano. Estos incendios están relacionados principalmente

con prácticas agrícolas, apertura de nuevos asentamientos rurales, limpia de cultivos, ganadería intensiva, la expansión de las fronteras agrícolas y la conversión de tierras (1). Otro motivo de menor relevancia, son los incendios ocasionados por los cazadores que utilizan este método para movilizar las presas, principalmente en las áreas de conservación (1).

El estudio de los incendios forestales recae en la importancia de sus efectos, si bien los incendios forestales tienen beneficios dentro de los ecosistemas, como la reducción de combustibles (pastos, hojarasca, hierbas, etc.), regulación de poblaciones, rebrote de pasto que sirve para la alimentación de los ganados y la fauna silvestre; después que ocurre un incendio 
forestal; es preocupante los efectos negativos tanto económicos, ambientales y de salud que causan. La ocurrencia de los incendios forestales se ha alterado, debido a factores socio económicos y climáticos, generándose una tendencia al aumento de los incendios ocurridos en las últimas décadas (2). Si bien los incendios forestales no siempre han sido como son hoy, hubo épocas en que fueron más intensos y severos que cualquier incendio en la historia de la humanidad, pero también hubo tiempos en los que su presencia fue mínima y en moderadas intensidades (3). Cada incendio "se comporta", es decir, evoluciona o progresa de forma distinta porque se desarrolla en un ambiente diferente. Además para la gestión, protección o restauración de las áreas afectadas por incendios forestales, supone una cuantificación de superficie, frecuencia, intensidad, severidad, extensión, distribución, estacionalidad de los incendios que requiere información de su localización, y que se logra con empleo de la teledetección, lo que requiere investigaciones a profundidad entorno a este problema.

\section{Efectos de los incendios forestales}

\section{Perdidas económicas}

Los incendios forestales generan gran impacto económico en el sector público y privado esto se manifiesta a una escala nacional que está ligado con la pérdida de productos forestales, de servicios, y los costos post-incendios como: costos de reforestación, de rehabilitación, incluidos suelos, ríos, etcétera; valores ecológicos, históricos, políticos, escénicos, y los costos del combate de incendios, que incluyen al personal y los recursos materiales. Pero la evaluación de las pérdidas económicas no es tan simple, requiere establecer valores económicos a la propiedad, los bienes y los servicios de las áreas forestales.

\section{Efectos en las cuencas hidrográficas}

Estos efectos van a depender a factores como la intensidad del incendio forestal, proporción del área quemada, distancia entre el área en la que ocurre el incendio forestal y la ubicación de la fuente de agua. En base a ello los efectos son: aumento de la escorrentía superficial que puede causar un aumento en la erosión, inundaciones, depósito de sedimentos; que conduce a la turbiedad, y contaminación de los ríos, (4) riesgo de inundaciones y de aterramientos muy superior al de las cuencas no quemadas (2). Disminución del tamaño de las gotas como la modificación en la estructura térmica de la atmósfera, llevan a una disminución de las precipitaciones en las regiones afectadas por aerosoles, una situación observada tanto en zonas afectadas por contaminación por quema de biomasa como por contaminación urbana. Por otra parte estos mismos efectos producen que, en los casos en los que se llegan a formar nubes precipitantes en regiones contaminadas, estas se producen a mayor altura y son mucho más violentas (1) todos estos efectos conducen a la alteración del ciclo hidrológico.

\section{Contaminación atmosférica}

Esta es una de las consecuencias de mayor impacto y preocupación. Más del $50 \%$ de emisiones en nuestro país proviene de la quema y deforestación de bosques (3). Las emisiones incluyen: gases de efecto invernadero como, $40 \%$ de dióxido de carbono (CO2), $16 \%$ de metano $(\mathrm{CH} 4)$ y $30 \%$ de óxido nitroso (N2O) de las emisiones antropogénicas totales. Gases como el monóxido de carbono (CO), metano (Ch4), monóxido y dióxido de nitrógeno (NO, NO2) son precursores de ozono troposférico. También se emiten gases que afectan el ozono estratosférico como el cloruro y bromuro de metilo $(\mathrm{CH} 3 \mathrm{Cl}, \mathrm{CH} 3 \mathrm{Br})$ (3). Además compuestos orgánicos persistentes como dioxinas y furanos $(1,3)$. Los aerosoles liberados contienen elementos metálicos producidos por el calentamiento de la vegetación y los suelos (14). Sus emisiones contribuyen a inyectar a la atmosfera grandes cantidades de gases de efecto invernadero y por tanto al aumento de la temperatura media del planeta. Gases como el CO, NOx, SO2, 
$\mathrm{HCN}$ y aerosoles provocan alteraciones en el balance radiactivo de la tierra, (3) toda la alteración de la química atmosférica conlleva a variaciones en el albedo y la alteración del clima.

\section{Efectos en la agricultura}

Ocasionan pérdidas importantes a granjeros y la comunidad local, dichos efectos son: Destrucción de equipos, maquinaria e instalaciones dañados por los incendios, muerte de animales domésticos (caballos, ganado caprino y vacuno), aparición de plagas y enfermedades en los bosques debilitados por los incendios forestales (5). Los incendios forestales disminuyen la producción y causan la mortalidad de especies de productos forestales no maderables importantes como las frutas, árboles medicinales y otros. Además afectan a los pequeños y grandes agricultores y ganaderos; por la quema de infraestructura tales como cercas, edificios y equipo, dando lugar a la reducción de capacidad de producción (6).

\section{Efectos sobre la biodiversidad}

Los efectos en la biodiversidad son: Perdida de la flora asociada al bosque, muchas veces formaciones vegetales primarias, afectaciones a la fauna y la muerte de numerosas especies autóctonas (2). Aumento de la vulnerabilidad de los bosques a los incendios recurrentes y los cambios en la composición del bosque y de la disponibilidad de frutos (6); que conlleva a la disminución de las especies de alimentación frugívora. Reducción de la tasa de crecimiento de los bosques supervivientes. La disminución de las áreas boscosas favorece el aumento de la radiación solar entrante, seca el interior del bosque, genera el aumento de la cantidad de material muerto y más capas de combustible en el suelo. Además cambios de la composición florística del bosque o comunidad vegetal, no proliferación de las especies exóticas zonas quemadas y destrucción de los paisajes naturales; reduciendo el valor recreativo del bosque (5).
Por otro lado, la actividad de la fauna disminuye drásticamente tras un incendio. Las aves nidificantes se eligen a menudo como indicadores de las consecuencias del fuego sobre la fauna (2). Los grupos más afectados son probablemente los reptiles, mientras que los animales terrestres con movilidad reducida están heridos o muertos, las especies de rápido movimiento también tienen problemas a medida que posiblemente tengan que competir por los recursos fuera de sus hábitats originales. Los individuos restantes pueden ser fácilmente cazados y sufren escasez de alimentos y la necesidad de cambiar la dieta (7). Estas perturbaciones del fuego reducen la abundancia de las comunidades de invertebrados, disminuyendo la disponibilidad de la densidad de la presa de algunas especies de aves. No obstante, los bosques y matorrales recientemente quemados suelen favorecer el desarrollo de pastos de calidad, lo que crea un buen hábitat para los hervivoros y aumenta el potencial cinegético (2).

\section{Efectos en el suelo}

Los incendios forestales afectan al suelo de diversas formas. En inicio existe un aumento de los cationes de cambio $(\mathrm{K}+, \mathrm{Mg}+2$, $\mathrm{Ca}+2$, etc.) en la solución del suelo, pero después ocurre perdidas por lavado que son muy grandes ya que disminuye la capacidad del complejo absorbente para retener nutrientes (4). El suelo al quedar desnudo, en tanto no se produzca la colonización vegetal post-incendio está expuesto a agentes erosivos como el agua y el viento. Dichos efectos erosivos se van a incrementar como consecuencia de la destrucción de la materia orgánica y de la estructura del suelo (4). Durante un incendio se pueden alcanzar temperaturas de $1200^{\circ}$ a $1400^{\circ} \mathrm{C}$, llegando la superficie del suelo a 1000 $\mathrm{C}$ por ello la microflora y la microfauna son destruidas casi en sus totalidad (4). Por otro lado el incendio incrementa el $\mathrm{pH}$ del suelo, debido a que las cenizas procedentes del incendio, contienen gran cantidad de carbonato potásico (CO3K2) (4). 


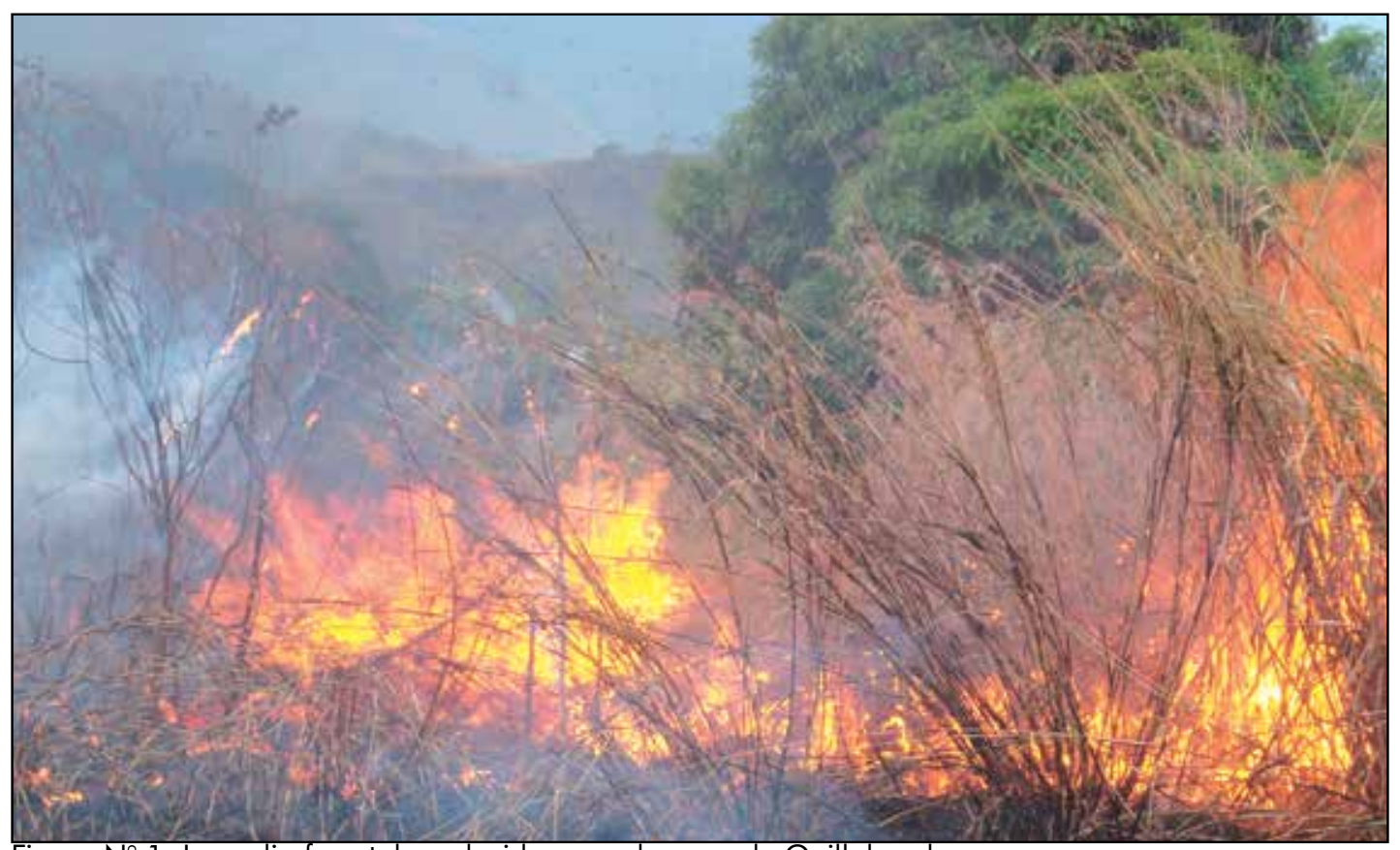

Figura $\mathrm{N}^{\circ}$ 1: Incendio forestal producido en un bosque de Quillabamba.

Fuente: Panared Noticias Quillabamba..

La alta severidad del fuego lleva a una reducción en el contenido de materia orgánica y por tanto un decrecimiento de la estabilidad de agregados y con ello el aumento de la densidad aparente (4), además de la pérdida de nitrógeno durante la combustión (4). El incendio afecta negativamente a la estructura del suelo y se producen pequeñas variaciones de la textura.

\section{Efectos en la salud}

Los incendios forestales exponen a los moradores cercanos a las zonas incendiadas; sobre todo a los niños; a un gran número de peligros relacionados al fuego, humo, estimulaciones psicológicas y los subproductos de la combustión de la madera, de los plásticos y de otros productos químicos que se están quemando. Durante la fase aguda de actividad del incendio forestal, lo más peligroso para los niños es el fuego y el humo (8). Existe el aumento de la incidencia de enfermedades respiratorias inducida por el humo, que normalmente se observan en el pico de la temporada de incendios, y que son responsables de un promedio de 9000 pacientes hospitalizados cada año durante la combustión de los incendios en la Amazonia.(6) Por otro lado se dan el cierre de escuelas en días de concentración de humo extremas, por lo cual los estudiantes pierden días de clases.

\section{Teledetección}

La teledetección es aquella técnica que permite adquirir imágenes e información de la superficie terrestres desde sensores instalados en plataformas especiales $(9,10)$. Los sensores son instrumentos susceptibles de detectar la señal electromagnética (radiación reflejada o emitida) que les llega de la tierra y la atmosfera y convertirla en una magnitud física que puede ser tratada y grabada. Los sensores convierten la señal electromagnética en un formato analógico (fotografía) o digital (imagen) (10). Las señales captadas por el sensor se amplifican, cuantifican y registran y pueden ser transmitidas a la tierra directamente a medida que son adquiridas o bien se almacenan en el satélite a la espera de su paso por una estación receptora (10).

Los datos de sensores remotos proporcionan una excelente base para monitorear los parámetros de interés para uso de los gestores e investigadores; como 
la biomasa, el estado de la vegetación, la ocurrencia de incendios activos y la delimitación de las áreas que se incendian, ubicación geográfica de cualquier punto de la imagen, (11) entre otras utilidades. Para llevar a cabo la observación del terreno, los sensores se ubican en plataformas, fijas o móviles: mástiles, grúas, globos, aviones, cohetes o satélites. En base a ello las características de la imagen registrada y de los datos que se obtienen dependen en gran medida de las propiedades del sensor utilizado y de la distancia al suelo desde la cual se realiza la adquisición de datos (10). Cada satélite y sensor tienen características especiales, pues fueron diseñados para cubrir necesidades específicas de información (5).

Los datos tienen en general diferente cobertura debido a la órbita del satélite, sus horarios de paso, el ancho de barrido transversal a la trayectoria, la sensibilidad de los algoritmos de detección.

\section{Uso de la teledetección en incendios forestales}

El empleo de teledetección satelital ha abierto al hombre, nuevas posibilidades para la detección temprana, mejor control y manejo de los incendios desencadenados en el medio forestal, lo cual resulta difícil y complejo utilizando los métodos tradicionales (2) y si bien las observaciones de campo siempre serán una parte vital del manejo del fuego, el tamaño de las zonas en cuestión son demasiado extensas - su ubicación es en zonas de difícil acceso y que a menudo significa que la observación de campo por sí sola no puede proporcionar suficiente información con exactitud y regularidad para proporcionar una base fiable (11). Por ello los datos de satélite son ampliamente empleados para la realización de estudios e investigaciones sobre el fuego ya que proporcionan información sobre la cobertura geográfica y frecuencia de los incendios. La detección de incendios forestales se basa en la detección de focos de calor. Los datos de focos de calor son básicamente puntos (coordenadas geográficas) que indican el centro de un pixel donde se detectó un foco de calor activo, en el momento en que un satélite hizo su paso por un área determinada (9).

\section{Gestión de incendios forestales}

Las investigaciones han demostrado que la teledetección puede proporcionar un apoyo muy útil para los gestores de incendios, ya que; considerar el manejo de incendios necesita de información en el contexto de un sistema de información para la gestión de incendios (5). El manejo del fuego es visto como una herramienta eficaz en la gestión de (a menudo) grandes extensiones de terreno (11). Dicho manejo por lo general comprende actividades destinadas a controlar la frecuencia, el área, la intensidad o el impacto de las actividades del fuego, ello se realizan en diferentes contextos institucionales, económicos, sociales, ambientales y geográficos, así como a diferentes escalas, desde la local hasta nacional (5). La gama de las actividades de manejo del fuego también varía considerablemente en función de los problemas de gestión que están en juego, así como los medios y la capacidad para actuar que estén disponibles. Cualquiera que sea la causa, la gestión eficaz de incendios requiere de información confiable sobre la cual basar las decisiones adecuadas y acciones, información que se requerirá en muchas etapas diferentes de una adecuada gestión (5) información que se obtiene usando adecuadamente tecnologías de teledetección.

\section{Ventajas de la teledetección}

La teledetección es una herramienta con mayor ventaja frente a otras técnicas. Los beneficios que proporcionan los datos de teledetección para el manejo del fuego son: que a menudo es menos caro $(5,3)$ y más rápido que la obtención de la misma información en el suelo en grandes áreas que permite la captura de datos a través de una gama más amplia del espectro electromagnético, que el ser humano es incapaz de ver, esto puede permitir la extracción de una gama más amplia de la información generada en la ocurrencia 
de los incendios (5). Las observaciones son espacialmente integrales, que cubreen grandes áreas del territorio, incluidas las zonas remotas y de difícil acceso por tierra, además que las observaciones son fiables, sistemáticas, objetivas y regulares, lo que permite actualizaciones frecuentes de la situación debido a que los satélites orbitan la tierra de forma continua. En base a ello la teledetección permite una detección temprana y un monitoreo aceptable de la evolución de los focos de calor.

\section{Satélites}

Son objetos artificiales en el espacio que dan vueltas alrededor de la tierra siguiendo una órbita específica. Son las plataformas más adecuadas para obtener una visión de regiones de gran extensión y, a diferencia de los cohetes, pueden permanecer mucho tiempo en órbita, permitiendo una visión constante de la tierra (10). Actualmente un gran número de satélites se encuentran proporcionando información aplicable a los trabajos de incendios y otro gran número de satélites se están diseñando (3). Pero solo se estudió a los más utilizados en el monitoreo de incendios forestales.
Tipos de satélites según el tipo de orbita:

\section{- Satélites geoestacionarios o geosincronos}

Se desplazan según una órbita ecuatorial, alta (aproximadamente 35 $900 \mathrm{Km}$ ). A esta distancia la velocidad orbital del satélite es la misma que la rotación de la tierra (15º de longitud por hora), por ello los satélites parecen estar fijos sobre un punto de la tierra y se mueven acompañado de la tierra en su movimiento. La órbita geoestacionaria es la que suelen utilizar los satélites de comunicación (10). El principal satélite de este tipo tenemos:

\section{Satélite GOES-12 (Geoestacionary} Operation Environmental Satellite)

Es un satélite geoestacionario (fijo sobre un punto de la tierra), de gran altura (35 $786 \mathrm{Km})(2,4)$. Desarrollado por la NASA principalmente para poder entregar imágenes meteorológicas en menor tiempo que las obtenidas mediante el satélite NOAA, (4) obtienen datos cada 15 minutos y sus datos son de

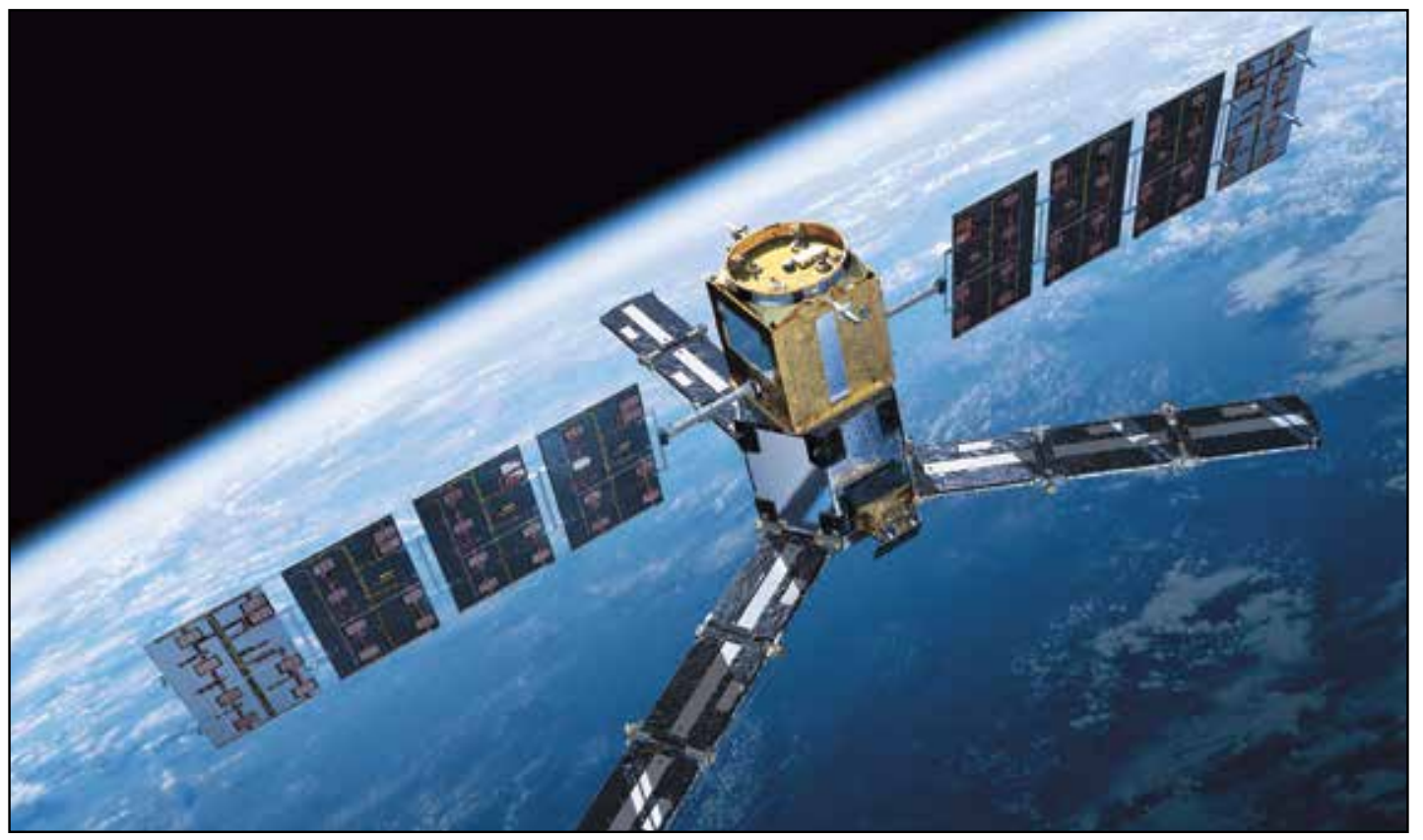

Figura $\mathrm{N}^{\circ}$ 2: Satélite geoestacional europeo SMOS

Fuente: $\mathrm{http} / /$ cronicadelespacio.blogspot.com 
rápida transmisión por lo cual es posible detectar de forma inmediata un fuego cuando se inicia (2). Su tarea, además de la adquisición de imágenes propias, es de retransmitir información de diferentes estaciones en todo el mundo, además contiene un sistema de monitoreo y triangulación de señales de emergencia en las frecuencias designadas internacionalmente (4). Es ampliamente utilizado en labores de detección de incendios, aunque tiene baja resolución espacial presenta una alta resolución temporal (2). Dada a la distancia a que se encuentra, la sensibilidad del sensor disminuye, por lo que tiene menor capacidad de identificación de fuego que los satélites de órbita polar que se encuentran a menores alturas (2). Para que los incendios sean detectados tienen que tener una temperatura mayor de $100 \stackrel{\circ}{C}(13)$.

Sensor GOES: El satélite GOES-12 cuenta también con varios instrumentos a bordo que permiten realizar mediciones atmosféricas, pero el instrumento que nos interesa es el que se lleva el mismo nombre. Tiene una resolución espacial de entre 2,5 y $4 \mathrm{~km}$. Provee imágenes de más del $60 \%$ de la superficie de la tierra (2). En varias investigaciones muestran en sus resultados preliminares que examinaron el patrón diurno de la distribución de incendios en la Amazonía, utilizando los datos del GOES, muestran una disminución del $20 \%$ en el incendio detectado frecuencia 15:30-18:30. (12).

\section{- Satélites helio síncronos}

Se sitúan en una forma heliosincrona, esto es, el plano de la órbita del satélite se mantiene fijo en relación al plano orbital de la tierra alrededor del sol. Estas orbitas solo son posibles entre los 300 y 1500 km de altitud. Para poder observar un área de la Tierra tan grande como sea posible, la inclinación de la órbita es casi de $90^{\circ}$, es decir, casi polar, de forma que la cobertura de la tierra visible por el satélite se extiende entre los dos polos. Su periodo de revolución es corto, inferior a 2 horas, y realizan al menos 12 vueltas alrededor de la tierra en 24 horas (10). La orbita está diseñada de tal forma que el paso del satélite por un lugar determinado se efectúa siempre a la misma hora local, es decir, el satélite cruza el ecuador siempre a la misma hora. Debido a su menor altitud la resolución espacial es del orden de metros. Sin embargo, esta menor altitud tiene como efecto que los satélites se vean frenados por la atmosfera terrestre, lo que obliga a corregir los parámetros de la órbita de forma periódica (10).

Dentro de este tipo de satélites tenemos a:

\section{Satélite NOAA}

Se encuentran en órbita desde octubre de 1978 (12). Son de órbita polar heliosincronica con una inclinación de 98,70 , de baja altura entre 833 a 870 $\mathrm{km}$ y de uso meteorológico. Proveen una imagen cada doce horas. Al tener una órbita heliosincronica este satélite pasa cada día por un mismo lugar a la misma hora. Por ser un satélite par presenta un nodo ascendente a las 19:30 y un nodo descendente a las 7:30. Ofrece todos los días datos globales $(10,12)$. La familia de satélites NOAA tienen ocho sensores a bordo, de los cuales a continuación solo describiremos el sensor utilizado.

Sensor AVHRR (Advanced Very High Resolution Radiometer): que está diseñado para proporcionar imágenes con una resolución espacial de 1,1 x $1,1 \mathrm{~km} 2$ en nadir $(4,9)$. La resolución espectral está dada por cinco bandas del espectro magnético(rojo, infrarrojo cercano y tres de infrarrojo térmico), la banda 1 ; con una región espectral en el rojo y un rango espectral de 0,58-0,68 $\mu \mathrm{m}$, la banda 2 ; con una región espectral en el infrarrojo cercano entre 0,72-1,10 $\mu \mathrm{m}$, la banda 3 con una región espectral en el infrarrojo medio y rango espectral de 3,55-3,93 $\mu \mathrm{m}$, la banda 4 y 5 con una región espectral en el infrarrojo térmico con un rango espectral de 10,3-11,3 $\mu \mathrm{m}$ 
y $11,5-12,5 \mu \mathrm{m}$ respectivamente $(4,12)$. Son las bandas 3 y 4 del sensor AVHRR los que comúnmente se utilizan en la identificación automática de incendios (9) aunque los dos primeros canales (1 y 2) son útiles para la identificación visual de la ocurrencia del fuego, lo que se define en algunos casos por la presencia de plumas de humo (12). Lo que le convierte en una herramienta adecuada para la teledetección regional y mundial de los incendios. Cada imagen provista por los satélites NOAA cubre una faja de aproximadamente $250 \mathrm{~km}$ de ancho por $400 \mathrm{~km}$ de longitud (12). La cobertura tan extensa en tan poco tiempo es la ventaja de utilización de los satélites NOAA (9).

El sensor AVHRR presenta una mayor resolución temporal $(9,13)$ y bajo coste el sensor AVHRR ha ganado un creciente interés para abordar asuntos medioambientales. Su amplia cobertura y baja resolución le permiten analizar las condiciones de la vegetación en periodos cortos de tiempo y escala global, lo que le hace muy idóneo para estudiar fenómenos muy dinámicos como la desertificación, la deforestación de zonas inaccesibles, o los incendios forestales de gran magnitud (9). Las limitaciones del sistema AVHRR para la vigilancia de los incendios son el bajo nivel de saturación de los sensores; confundiendo incendios con terrenos desnudos capaces de presentar temperaturas de esa magnitud, especialmente en áreas desérticas durante el verano, o bien nubes muy brillantes (17). Pero a este inconveniente se da solución con el uso de datos nocturnos que ofrecen importantes posibilidades para disminuir e incluso eliminar la confusión. El uso de imágenes nocturnas elimina dos inconvenientes principales a) la saturación del canal 3 por emisión de calor de superficies no incendiadas, y b) el problema derivado de la reflexión solar durante el día (12).

\section{Satélite TERRA y AQUA}

Ambos satélites son de órbita polar heliosincronica, es decir alineados al sol, son de baja altura y de múltiple uso. Lanzados en diciembre de $1999(4,12)$. El satélite TERRA desciende a través del ecuador a las 10:30 de la mañana (3) y el satélite AQUA asciende a través del ecuador a las 1:30 de la tarde (4), lo cual permite observar los fenómenos de la tierra, por la mañana con TERRA y por la tarde con AQUA (13) Los satélites AQUA y TERRA son parte de la Earth Science Enterprise de la NASA, tienen canales diseñados específicamente para la detección de fuego y una cobertura global continua de cada 1 a 2 días (2).

Sensor MODIS (Moderate-Resolution Imaging Spectroradiometer): cuenta con 36 canales 0 bandas, a distintas resoluciones espaciales y ámbitos del espectro electromagnético $(4,12)$. Las dos primeras bandas tienen $250 \times 250$ $\mathrm{m} 2$ de resolución espacial cubriendo el rojo e infrarrojo cercano, las 5 bandas siguientes ofrecen $500 \times 500 \mathrm{~m} 2$ de resolución espacial y cubren el espectro visible y diversas bandas de la onda corta infrarroja $(4,2)$. El resto de las bandas tienen una resolución espacial de $1 \times 1 \mathrm{Km} 2$ cubriendo principalmente el espectro del infrarrojo cercano, el infrarrojo medio y térmico (6 bandas entre 10,78 y $14,36 \mu \mathrm{m})$. Cuenta con un área de barrido de $2300 \mathrm{~km}$, lo que le facilita una cobertura prácticamente diaria de toda la tierra $(4,2)$. Este sensor es de mediana resolución (13) y se utiliza operativamente para transmitir información de incendios activos en tiempo real, siendo de especial utilidad en aquellos países que no tienen información de detecciones terrestres (2). La exactitud de geolocalizacion y las características radiométricas más convenientes permiten la elaboración de productos de mayor precisión sobre los fuegos activos y las áreas quemadas, conocer los ciclos diarios de los incendios en todas las regiones del planeta, velocidad de propagación así como obtener datos útiles para realizar la representación espacial de los fuegos (3). Además proporcionan datos para estudiar aerosoles, partículas, y los 
productos de la combustión de biomasa (12).

\section{CONCLUSIONES}

- En las últimas décadas los incendios forestales se han convertido en un problema ambiental de gran preocupación a nivel mundial, sobre todo en los países con gran riqueza forestal; esto debido a que se ha incrementado la ocurrencia de estos, además de los impactos económicos, ecológicos y de salud que causan.

- Uno de los impactos negativos y de mayor preocupación que generan los incendios forestales es la contaminación atmosférica; los incendios forestales emiten más del $50 \%$ de las emisiones antropogenicas. Dicha contaminación atmosférica conlleva a otros problemas ambientales como la alteración del ciclo hidrológico, calentamiento global, entre otros.

- Los impactos en la flora, fauna, suelo y fuentes de agua, aumento de enfermedades respiratorias, perdidas económicas son otros de los impactos que nos hacen ver que el problema de los incendios forestales debe de ser estudiado para en la medida posible evitar su ocurrencia.

- La teledetección es una herramienta con grandes beneficios para la gestión de los incendios forestales, en muchos países existe una exitosa aplicación de esta herramienta, pero que en nuestro país aún no se ha aprovechado las ventajas de esta herramienta que sin duda nos brindaría una base de información, datos e incluso imágenes acerca de la ocurrencia de los incendios forestales; incluso de las zonas más remotas y de difícil acceso.

- La teledetección se lleva a cabo por medio de la detección de focos de calor; que son coordenadas geográficas con la ubicación de un incendio forestal.

- Existen diversos satélites que en la actualidad circulan la tierra, obtienen y transmite información de diversos aspectos. Algunos satélites y sus respectivos sensores presentan características específicas y particulares que le permiten la detección de incendios forestales con una alta confiabilidad, la elección de ellos va depender de las necesidades del investigador o gestor del incendio forestal.

- En la actualidad el estado peruano ha desarrollado leyes y reglamentos que priorizan la defensa contra los incendios forestales para evitar y reducir los daños ocasionados por los incendios forestales dentro de los cuales se encontraría la creación e implementación del "Sistema Nacional de Prevención y Control de Incendios Forestales" que involucra a diversas autoridades e instituciones nacionales, regionales $y$ locales y a la población en general, tal como lo establece el artículo 4 de la ley forestal y de fauna silvestre, que en la actualidad no se realiza, además del incumplimiento del DS $\mathrm{N}^{\circ} 014$ que establece el desarrollo del "Plan Nacional de Prevención y Control de Incendios y Plagas Forestales" que lamentablemente este plan tampoco se ha implementado hasta la fecha. Por otro lado en el Perú no existe investigaciones con una base de datos e información confiable acerca de la ocurrencia de los incendios forestales que ayudarían en gran medida para que las autoridades puedan cumplir las normas antes mencionadas.

\section{Agradecimientos}

Por su apoyo económico para la formulación de mi tesis universitaria a, la National Academy of Sciences de los Estados Unidos (NAS) y a la United State Agency for International Development (USAID). Asimismo, a la dirección de investigación de la Universidad Continental en Perú por la asesoría académica científica. 


\section{REFERENCIAS BIBLIOGRÁFICAS}

1. Mielnicki DM, Canziani PO. Quema de biomasa en Sudamérica: impactos regionales y globales.Argentina:2-6.

2. Sedeño EM, Setzer AW. Sistema para la detección y monitoreo de incendios en la vegetación con el empleo de la teledetección - pronóstico de peligro a corto y mediano plazo. In: XV Congreso mexicano de meteorología. Mexico.2006:21-46.

3. Crutzen PJ, Andreae MO. Biomass burning in the tropics: impact in atmospheric chemistry and biogeochemical.Science.1990; 250: 1669. 1678.

4. De las heras JI, Martinez JS, Herranz JS. Impacto ecológico de los incendios forestales. Universidad de Castilla. 2001.

5. Flasse SP, Trigg SN, Ceccato $\mathrm{PN}$, Perryman AH, Hudak AT, Thompson $M W$, etal. Remote sensing of vegetation fires and its contribution to a fire management information system In: Goldammer JG, De Ronde C, editors. Wildland fire management handbook for sub-Sahara Africa. Global fire monitoring Center. 2004:158-185.

6. Schroeder W, Alencar A, Arima E, Setzer A. The spatial distribution and interannual variability of fire in Amazonia. In Washington DC American Geophysical Union Geophysical. 2009; 186:43-60.

7. Cardoso MF, Hurtt GC. Potential for advancing fire research in Amazonia by integrating ground-based, remotesensing and modeling studies for the region. In: Gomez E, Alvarez K, editors. Forest Fires: Detection, Suppression and Prevention. Brazil. 2009:2-14.

8. James M, Seltzer MD, Miller MD, Seltzer MA. Riesgos a la Salud de los Niños por los Incendios Forestales - Fase Aguda. Universidad de California, San Francisco. 2007.

9. Chuvieco E. Fundamentos de teledetección espacial. Segunda edición. Madrid: RIALP SAC; 1995.
10. José Sobrino. Teledetecciòn. Universitat de valencia editor.2001.pag 33-53

11. Bond JW, Midgley JJ. Fire and Plants. Population and Community Biology. London. $1996 ;(14): 263$.

12. Justice CO, Malingreau JP, Setzer AW. Satellite remote sensing of fires: potential and limitations. In: Dahlem Workshop Reports Berlin. Alemania. 1992:15-20.

13. Lillesand TM, Ralph WK, Chipman JW. Remote sensing and image interpretation. Quinta edicion. Wiley J, Sons editors. 2004. 